\title{
Computed tomographic pulmonary angiography and pulmonary embolism: predictive value of a d-dimer assay
}

\author{
Patricia Deonarine ${ }^{1 *}$, Carl de Wet $^{2}$ and Alistair McGhee ${ }^{3}$
}

\begin{abstract}
Background: Computed tomographic pulmonary angiography (CTPA) is increasingly being used as first investigation for suspected pulmonary embolism (PE). The investigation has high predictive value, but is resource and time intensive and exposes patients to considerable radiation. Our aim was to assess the potential value of a negative $d$-dimer assay to exclude pulmonary emboli and reduce the number of performed CTPAs.

Methods: All CTPAs performed in a Scottish secondary care hospital for a fourteen month period were retrospectively reviewed. Collected data included the presence or absence of $\mathrm{PE}, \mathrm{d}$-dimer results and patient demographics. PE positive CTPAs were reviewed by a specialist panel.

Results: Pulmonary embolisms were reported for 66/405 (16.3\%) CTPAs and d-dimer tests were performed for 216 (53\%). 186/216 (86\%) patients had a positive and 30 (14\%) a negative d-dimer result. The panel agreed 5/66 (7.6\%) false positive examinations. The $d$-dimer assay's negative predictive value was 93.3\% (95\% Cl=76.5\%-98.8\%) based on the original number of positive CTPAs and $100 \%(95 \% \mathrm{Cl}=85.9 \%-100 \%)$ based on expert review. Significant nonPE intrapulmonary pathology was reported for 312/405 (77.0) CTPAs, including 13 new diagnoses of carcinoma.

Conclusions: We found that a low d-dimer score excluded all pulmonary embolisms, after a further specialist panel review identified initial false positive reports. However, current evidence-based guidelines still recommend that clinicians combine a d-dimer result with a validated clinical risk score when selecting suitable patients for CTPA. This may result in better use of limited resources, prevent patients being exposed to unnecessary irradiation and prevent potential complications as a result of iodinated contrast.
\end{abstract}

Keywords: Pulmonary embolism, D-dimer, CTPA (tomography)

\section{Background}

Pulmonary embolism (PE) is associated with substantial morbidity and mortality. In the US more than 500000 patients per year are diagnosed with pulmonary emboli, resulting in approximately 200000 deaths [1,2]. In England and Wales there are around 65000 cases of pulmonary emboli annually amongst hospitalized patients. The prevalence of unsuspected pulmonary embolism at post-mortem is $3-8 \%$, figures that have changed little over three decades. The implication is

\footnotetext{
* Correspondence: patdeo75@yahoo.co.uk

'Radiology Department, Glasgow Royal Infirmary, 84 Castle Street, Glasgow G4 OSF, UK

Full list of author information is available at the end of the article
}

that the true number of cases may be substantially higher than is currently being diagnosed [3].

The presentation, symptoms and clinical signs of pulmonary embolism varies widely between patients [4]. Clinical suspicion invariably requires further investigation to confirm or exclude PE. In Scotland, computed tomographic pulmonary angiography (CTPA) is increasingly used as the first and only investigation for this purpose [5]. This is because of greater availability and reported overall sensitivity (89-100\%) of helical CT, but exposes patients to substantial ionizing radiation [6-8]. A recent review by Davies et al. found that iatrogenic radiation exposure has significant risks which are often overlooked, while it was estimated that $30 \%$ of computed tomography tests may be unnecessary [9].
C Biomed Central

(c) 2012 Deonarine et al; BioMed Central Ltd. This is an Open Access article distributed under the terms of the Creative Commons Attribution License (http://creativecommons.org/licenses/by/2.0), which permits unrestricted use, distribution, and reproduction in any medium, provided the original work is properly cited. 
D-dimer assays have low specificity, but high sensitivity and negative predictive value in most patients with suspected thromboembolism, and may be an alternative first investigation to CTPA [10]. D-dimers are degradation products of cross linked fibrin and are considered the best laboratory markers of coagulation activation [11,12]. They are commonly elevated in patients with recent surgery, malignancy or infection $[3,13]$. As a result the diagnostic value of $\mathrm{d}$-dimer assays is higher for ambulatory patients compared with those in hospital [14].

Our main aim was to assess the potential value of a negative $d$-dimer assay to exclude pulmonary emboli and reduce the number of performed CTPAs. A further aim was to describe incidental but significant intrapulmonary CTPA findings that may have accounted for patients' clinical presentations.

\section{Results}

A total of 416 CTPAs were performed during the study period. 11/416 (2.6\%) CTPAs were reported as indeterminate or inconclusive and excluded from further analysis. 'Technical factors', for example inadequate contrast opacification of the pulmonary arterial tree, were stated as the reason for inconclusive studies. Of the remaining 405 CTPA studies, 226 (55.8\%) were performed for female and 179 (44.2\%) for male patients. The mean age of all patients was 63 years (range 20-95 and standard deviation \pm 17.2)

A diagnosis of pulmonary embolism was reported for $66(16.3 \%)$ of the remaining 405 CTPAs. The expert panel unanimously agreed that five $(7.6 \%)$ of these were false positive examinations. The positive predictive value of CTPA for pulmonary embolism in this study was 92.4\% (95\% CI = 82.5\%-97.2\%).

The numbers of performed d-dimer assays, d-dimer results and CTPAs positive for pulmonary embolism are shown in Figure 1. Of the 216 (53.3\%) patients who had a d-dimer assay performed, $186(86 \%)$ had a positive and $30(14 \%)$ a negative d-dimer result. There were initially two positive CTPAs with negative d-dimer results. Both of these were unanimously judged to be negative CTPAs (false positives) by the panel. The d-dimer assay's negative predictive value was $93.3 \%$ (95\% CI = 76.5\%-98.8\%) based on the original number of positive CTPA reports and $100 \%$ (95\% CI $=85.9 \%-100 \%)$ based on the panel's review. The d-dimer assay and CTPA sensitivity, specificity and predictive values are shown in Table 1 . The complete data set is available as an additional file 1 (see CTPA ddimer.xls).

The main anatomical sites of pulmonary embolism are shown in Table 2. Sub segmental embolisms were least common, being described in $4(6 \%)$ of cases. The main findings of the CTPAs considered for analysis are shown in Table 3. Significant non-PE intrapulmonary pathology was

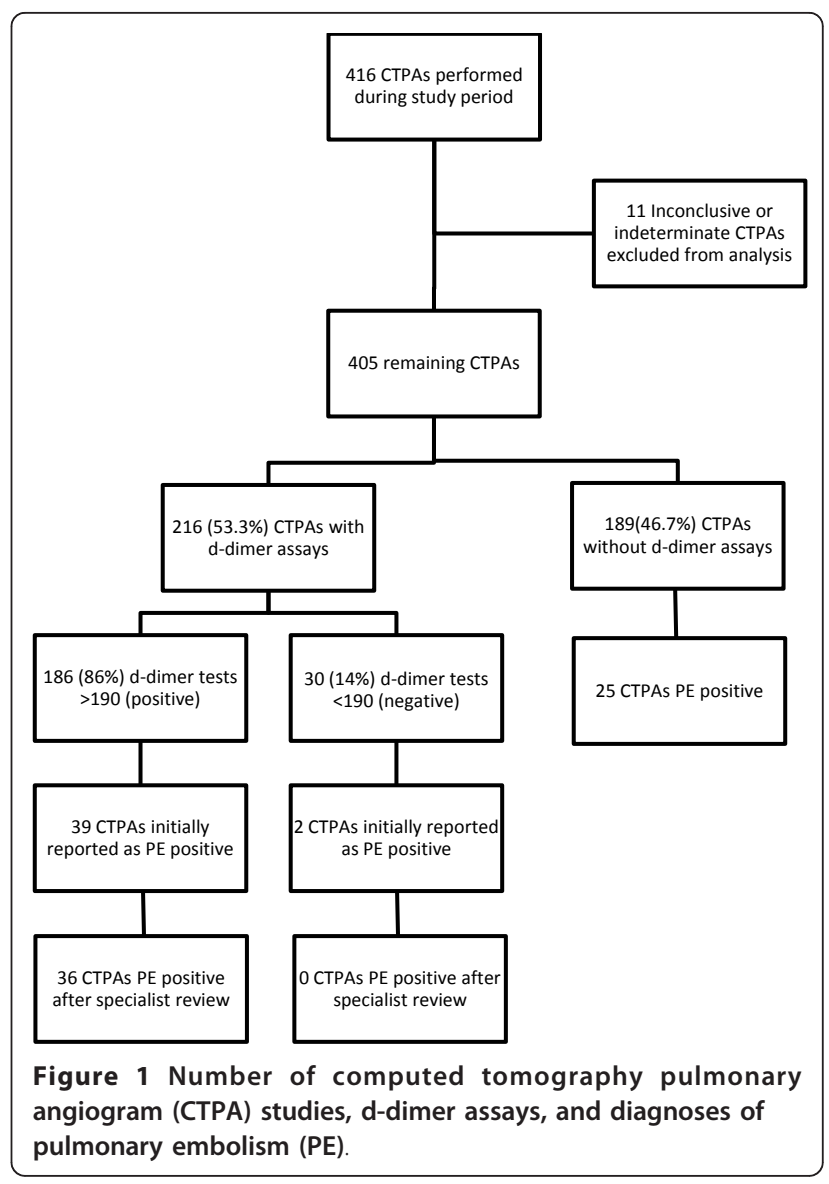

reported for 312/405 (77.0) CTPAs. The most common reported abnormalities were pleural effusion (16.5\%), bronchiectasis (10.6\%) and consolidation (7.9\%). There were 13 (3.2) new diagnoses of unsuspected carcinoma. Of the 66/405 (16.3) CTPAs reported as pulmonary embolism, 52 patients had significant additional pulmonary pathology.

\section{Discussion}

\section{Main findings}

The study's main aim was to assess the potential value of a negative d-dimer assay to exclude pulmonary emboli and reduce the number of performed CTPAs. We found a low (negative) d-dimer score to have a very high negative predictive value, but that it did not exclude all pulmonary emboli based on the initial CTPA reports. However, a low d-dimer score did exclude all pulmonary emboli after a further specialist panel review identified false positive reports.

\section{Comparison with existing literature}

Our findings are comparable to other studies in various health care settings. Dunn et al. reported a negative predictive value for $\mathrm{d}$-dimer assay of $99.6 \%$ (95\% CI $=98.7$ $>99.9 \%$ ) and suggested that negative results could help 
Table 1 D-dimer assay and CTPA sensitivity, specificity and predictive values (95\% confidence intervals)

\begin{tabular}{lccc}
\hline & \multicolumn{2}{c}{ Results based on number of positive CTPA results } & \multicolumn{1}{c}{ CTPA } \\
\cline { 2 - 4 } & $\mathbf{N}=\mathbf{4 1}$ (initial results) & $\mathbf{N}=\mathbf{3 6}$ (results after specialist review) & $\mathbf{N}=\mathbf{4 0 5}$ \\
\hline Sensitivity & $95.1(82.2-99.2)$ & $100(88.0-100)$ & $\times$ \\
\hline Specificity & $16.0(11.1-22.5)$ & $16.7(11.7-23.1)$ & $98.5(96.4-99.5)$ \\
\hline Positive predictive value & $21.0(15.5-27.7)$ & $19.4(14.1-25.9)$ & $92.4(82.5-97.2)$ \\
\hline Negative predictive value & $93.3(76.5-98.8)$ & $100(85.9-100)$ & $\times$ \\
\hline
\end{tabular}

to reduce the number of performed CTPAs [15]. More recently, Eng et al. and Hirai et al. concluded that a ddimer test alone was suitable for screening patients with a clinical suspicion of PE $[16,17]$. However, a number of case reports have questioned whether a negative $d$ dimer result alone is sufficient to exclude pulmonary embolisms [18]. There is compelling evidence that a negative $d$-dimer result can effectively exclude a PE when it is combined with a low pretest clinical probability score. Current best practice clinical guidance advises combining the d-dimer result with a validated tool-for example the Wells or Geneva rule-which allow risk to be quantified in a structured manner [19-22].

A further study aim was to describe incidental but significant intrapulmonary CTPA findings that may have accounted for patients' clinical presentations. Significant pathological findings were reported for the vast majority of CTPA studies. The number of CTPA reports which described previously undiagnosed malignancies was substantially higher than reported by Kino et al. [23]. These significant non-PE findings had clinical relevance for some patients and may subconsciously lead clinicians to rationalize CTPA requests. However, CTPA has certain technical limitations that reduce its potential value in assessing non-PE pathology and cannot be considered a screening tool.

The anatomical distribution of pulmonary emboli we found was comparable to that reported by Sohns et al. [24]. The majority of thrombi were diffusely distributed between the main pulmonary trunk, lobar and segmental arteries with only a small minority involving the subsegmental vessels. All of the false positive CTPAs were

Table 2 Classification of pulmonary emboli according to anatomical site

\begin{tabular}{lll}
\hline & No* $^{*}$ & (\%) \\
\hline Large main trunk & 21 & $(32)$ \\
\hline Lobar artery & 20 & $(30)$ \\
\hline Segmental & 21 & $(32)$ \\
\hline Sub-segmental & 4 & $(6)$ \\
\hline Total & $\mathbf{6 6}$ & $(100)$ \\
\hline
\end{tabular}

* Each PE positive case was included only once in the classification according to the largest thrombosed vessel reported initially reported as 'small, sub-segmental pulmonary embolism'. The expert panel's opinion was that in these cases small lymph nodes or veins adjacent to sub-segmental arteries had typically been misinterpreted as filling defects. It is possible that a substantial number of sub-segmental pulmonary emboli may be false positive as a result of CTPAs being interpreted in a single plane.

\section{Strengths and limitations}

Our findings are based on a substantial sample and an additional independent panel that reviewed CTPA studies to identify false positive results. We also identified the anatomical distribution of emboli and additional intrapulmonary pathology that may have accounted for the patients' symptoms. The study has a number of limitations: CTPA findings were not linked with clinical outcomes; validated tool such as the Geneva or Wells rule was not used or recorded by clinicians requesting CTPAs; other imaging, for example doppler ultrasound, chest $\mathrm{x}$-rays and ventilation perfusion scans were not considered; and more modern alternatives to the 16slice CT scanner used in our study may have improved diagnostic accuracy.

\section{Implication and future research}

There are clinical guidelines for health care workers investigating a patient suspected of having a PE [19-22]. It is recommended that a clinical probability assessment and d-dimer value should be combined and used to quantify the patient's risk of PE as low, moderate or high. CTPAs are only indicated for those patients judged to be at moderate or high risk. This approach is seldom used in practice, resulting in unnecessary CTPAs being performed. This is an inefficient use of limited time and resources and expose patients to avoidable irradiation and potential complications of iodinated contrast $[15,18]$. Further research is required to better understand the challenges in promoting and implementing the routine use of clinical risk stratification for ambulatory patients with suspected PE.

\section{Conclusions}

A low d-dimer score had a very high negative predictive value, but did not exclude all pulmonary embolisms 
Table 3 The main findings of 405 CTPAs performed between 01/06/2008 and 31/07/2009 that met inclusion criteria

\begin{tabular}{lcc}
\hline Main CTPA finding & No (\%)* & No (\%)* \\
\hline No pathology & & $103(25.4)$ \\
\hline Pulmonary embolism (PE) & & (16.3) \\
\hline$\cdot$ PE only & $14(3.5)$ & $32(12.8)$ \\
\hline$\cdot$ PE with additional abnormal finding (included below) & & $312(77.0)$ \\
\hline Significant non-PE intrapulmonary pathology & $67(16.5)$ & $32(7.9)$ \\
\hline$\cdot$ Pleural effusion & $27(6.7)$ & $13(3.2)$ \\
\hline$\cdot$ Consolidation & $22(5.4)$ & $13(3.2)$ \\
\hline$\cdot$ Effusion and consolidation & $43(10.6)$ \\
\hline$\cdot$ Carcinoma & $7(1.7)$ \\
\hline$\cdot$ Lobar collapse & $88(21.7)$ \\
\hline$\cdot$ Lymphadenopathy
\end{tabular}

*Some CTPAs had more than one main finding reported. The number and percentage is the proportion of the 405 CTPAs with that specific finding

based on the initial CTPA reports. However, a low ddimer score did exclude all pulmonary embolisms after a further specialist panel review identified false positive reports. A practical and evidence-based approach is to combine a d-dimer result with a validated clinical risk score to help select suitable patients for CTPA. This may result in better use of limited resources, prevent patients being exposed to unnecessary irradiation and prevent potential complications as a result of iodinated contrast.

\section{Availability of supporting data}

The data set supporting the results of this article is included within the article and its additional file 1.

\section{Methods}

All CTPAs that had been performed at a District General Hospital (DGH) in Lanarkshire, Scotland, in the fourteen month period from $1^{\text {st }}$ June 2008 to $31^{\text {st }}$ July 2009 were identified and retrospectively reviewed on the Hospital Information System (HIS). This sample included CTPAs requested for hospitalized and ambulatory patients. Patients were considered ambulatory if they had been referred from the accident and emergency department or from medical, surgical and oncology out-patient units. An indeterminate or inconclusive CTPA report was the only exclusion criterion.

All PE positive studies were reviewed independently by a panel consisting of three radiologists with an interest in this area who interpreted the CTPAs using axial, coronal and sagittal reformats. The initial CTPA reports were judged to be false positive only if all three panel members agreed that the study did not show sufficient evidence of PE. Data were collected for presence or absence of PE and the type of PE, whether a d-dimer assay was performed and the d-dimer result if applicable. Patients' age and gender and other reported pathological intra thoracic findings were also collected. Patient identifiers were removed and data were entered in an Excel spreadsheet. The data were exported to SPSS version 17.0 for calculation of descriptive statistics.

The d-dimer assay and CTPA sensitivity, specificity and positive and negative predictive values were calculated with $95 \%$ confidence intervals. The d-dimer assay values were calculated twice, using the initial number of positive CTPA results, and then the revised number of positive CTPA results as determined by the specialist panel. Pulmonary emboli were classified according to their anatomical distribution. Each case was included only once and grouped according to the largest thrombosed vessel reported.

\section{CTPA}

All CTPA studies were performed using a Toshiba Aquillon 16 slice CT scanner with slice thickness set at $1 \mathrm{~mL}$.

\section{D-dimer}

Four different types of d-dimer assay formats are currently available: enzyme linked immunosorbent assay (ELISA), whole blood erythrocyte agglutination assay (SimpliRED), semiquantitative latex agglutination assays (Accuclot, Trinity Biotech, Bray) and immunochromatographic/quantitative immunoturbidimetric assays. ELISA is considered the gold standard for the determination of d-dimer concentration. It is a highly sensitive test but is time consuming and not suitable for individual patient testing. The Accuclot d-dimer assay is less sensitive, but 
suitable for individual patient testing [25-27]. The Trinity Amax Accuclot d-dimer assay-a semi quantitative latex agglutination assay-was used during the study period. A d-dimer value $\geq 190 \mathrm{ng} / \mathrm{mL}$ was considered positive (high) and $<190 \mathrm{ng} / \mathrm{mL}$ negative (low) in accordance with local guidelines and the recommendation of the Accuclot d-dimer assay manufacturer.

\section{Additional material}

Additional file 1: The complete data set is available as a Microsoft Excel spreadsheet and can be downloaded as an additional file (CTPA ddimer.xIs)

\section{Acknowledgements}

We would like to thank the PACS team at Monklands DGH Lanarkshire, the Biochemistry department for access to laboratory records to review d-dimer values and Drs. Julien Guse and Tamas Schilzer.

\section{Author details}

${ }^{1}$ Radiology Department, Glasgow Royal Infirmary, 84 Castle Street, Glasgow G4 OSF, UK. ${ }^{2}$ General practitioner and research fellow, NHS Education for Scotland, Glasgow, UK. ${ }^{3}$ Radiology consultant, Monklands district general hospital, Lanarkshire, UK.

\section{Authors' contributions}

PD helped to design the study, collected the data and helped to prepare the manuscript. CdW coded and analyzed the data and helped to prepare the manuscript. AM had the original idea for the study, was one of the 'expert' reviewers and reviewed the final manuscript. All authors read and approved the final manuscript.

\section{Competing interests}

The authors declare that they have no competing interests.

Received: 24 November 2011 Accepted: 17 February 2012

Published: 17 February 2012

\section{References}

1. Dismuke S, Wagner E: Pulmonary embolism as a cause of death. The changing mortality in hospitalized patients. JAMA 1986, 255:2039-2042

2. Horlander K, Minnino D, Leeper K: Pulmonary embolism mortality in United States, 1979-1998: an analysis using multiple-cause mortality data. Arch Intern Med 2003, 163(1711):1717.

3. Torbicki A, Perrier A, Konstantinides S, Agnelli G, Galie N, Pruszczyk P, et al: Guidelines on the diagnosis and management of acute pulmonary embolism. Eur Heart J 2008, 29:2276-2315.

4. Courtney DM, Kline JA, Kabrhel C, Moore CL, Smithline HA, Nordenholz KE, et al: Clinical features from the history and physical examination that predict the presence or absence of pulmonary embolism in symptomatic emergency department patients: results of a prospective, multicenter study. Ann Emerg Med 2010, 55(4):307-315, e1.

5. O'Neill JM, Wright L, Murchison JT: Helical CTPA in the investigation of pulmonary embolism: a 6-year review. Clin Radiol 2004, 59(9):819-825.

6. Rathbun S, Raskob G, Whitsett T: Sensitivity and specificity of helical computed tomography in the diagnosis of pulmonary embolism: a systematc review. Ann Intern Med 2000, 32:227-232.

7. de Monye W, Pattynama P: Contrast enhanced spiral computed tomography of the pulmonary arteries: an overview. Semin Thromb Hemost 2001, 27:33-39.

8. O'Neill J, Murchison JT, Wright L, Williams J: Effect of the introduction of helical $\mathrm{CT}$ on radiation dose in the investigation of pulmonary embolism. Br J Radiol 2005, 78(925):46-50

9. Davies HE, Wathen CG, Gleeson FV: Risks of exposure to radiological imaging and how to minimise them. BMJ 2011, 342:589-593.
10. Ginsberg JS, Wells PS, Kearon C, Anderson D, Crowther M, Weitz Jl, et al: Sensitivity and specificity of a rapid whole blood assay for $D$ dimer in the diagnosis of pulmonary embolism. Ann Intern Med 1998, 129:1006-1011.

11. Kroneman $\mathrm{H}$, Nieuwenhuizen W, Knot EA: Monoclonal antibody-based plasma assays for fibrin (ogen) and derivates, and their clinical relevance. Blood Coagul Fibrinolysis 1990, 1:91-111.

12. Sie $P$ : The value of laboratory tests in the diagnosis of venous thromboembolism. Haematologica 1995, 80(2 Suppl):57-60.

13. Goldhaber SZ, Simons GR, Elliot CG, Haire WD, Toltzis R, Blacklow SC, et al Quantitative plasma D dimer levels among patients undergoing pulmonary angiography for suspected pulmonary embolism. JAMA 1993, 270:2819-2822

14. Schrecengost JE, LeGallo RD, Boyd JC, Moons K, Gonias SL, Rose CE, et al: Comparison of diagnostic accuracies in outpatients and hospitalized patients of D-dimer testing for the evaluation of suspected pulmonary embolism. Clin Chem 2003, 49:1483-1490.

15. Dunn KL, Wolf JP, Dorfman DM, Fitzpatrick P, Baker JL, Goldhaber SZ: Normal D-dimer levels in emergency department patients suspected of acute pulmonary embolism. J Am Coll Cardiol 2002, 40(8):1475-1478.

16. Eng CW, Wansaicheong G, Goh SK, Earnest A, Sum C: Exclusion of acute pulmonary embolism: computed tomography pulmonary angiogram or D-dimer? Singapore Med J 2009, 50(4):403-406.

17. Hirai LK, Takahashi JM, Yoon HC: A prospective evaluation of a quantitative D-dimer assay in the evaluation of acute pulmonary embolism. J Vasc Interv Radiol 2007, 18(8):970-974

18. Breen ME, Dorfman M, Chan SB: Pulmonary embolism despite negative ELISA D-dimer: a case report. J Emerg Med 2009, 37(3):290-292

19. Wells PS, Anderson DR, Rodger M, Stiell I, Dreyer JF, Barnes D, et al: Excluding pulmonary embolism at the bedside without diagnostic imaging: management of patients with suspected pulmonary embolism presenting to the emergency department by using a simple clinical model and D dimer. Ann Intern Med 2001, 135:98-107.

20. Subramaniam RM, Chou T, Swarbrick M, Karalus N: Pulmonary embolism: accuracy and safety of a negative CT pulmonary angiogram and value of a negative D-dimer assay to exclude CT pulmonary angiogramdetectable pulmonary embolism. Australas Radiol 2006, 50(5):424-428.

21. Gupta RT, Kakarla RK, Kirshenbaum KJ, Tapson VF: D-dimers and efficacy of clinical risk estimation algorithms: sensitivity in evaluation of acute pulmonary embolism. AJR Am J Roentgenol 2009, 193(2):425-430.

22. Gibson NS, Sohne M, Kruip MJ, Tick LW, Gerdes VE, Bossuyt PM, et al: Further validation and simplification of the Wells clinical decision rule in pulmonary embolism. Thromb Haemost 2008, 99(1):229-234.

23. Kino A, Boiselle PM, Raptopoulos V, Hatabu H: Lung cancer detected in patients presenting to the Emergency Department studies for suspected pulmonary embolism on computed tomography pulmonary angiography. Eur J Radiol 2006, 58(1):119-123.

24. Sohns C, Amarteifio E, Sossalla S, Heuser M, Obenauer S: 64-Multidetectorrow spiral $C T$ in pulmonary embolism with emphasis on incidental findings. Clin Imaging 2008, 32(5):335-341.

25. Lee AY, Ginsberg JS: The role of D-dimer in the diagnosis of venous thromboembolism. Curr Opin Pulm Med 1997, 3(275):279.

26. Perrier A, Desmarais S, Miron M, de Moerloose P, Lepage R, Slosman D, et al: Non-invasive diagnosis of venous thromboembolism in outpatinets. Lancet 1999, 353(9148):190-195.

27. Bernardi E, Prandoni P, Lensing AWA, Agnelli G, Guazzaloca G, Scannapieco G, et al: D-dimer testing as an adjunct to ultrasonography in patients with clinically suspected deep vein thrombosis: prospective cohort study. BMJ 1998, 317:1037-1040.

doi:10.1186/1756-0500-5-104

Cite this article as: Deonarine et al:: Computed tomographic pulmonary angiography and pulmonary embolism: predictive value of a d-dimer assay. BMC Research Notes 2012 5:104. 